\title{
Maxillary protraction using customized mini-plates for anchorage in an adolescent girl with skeletal Class 111 malocclusion
}

\author{
Shuran Liang $\mathbb{1 0}$ \\ Xianju Xie \\ Fan Wang \\ Qiao Chang \\ Hongmei Wang \\ Yuxing Bai (1)
}

Department of Orthodontics, School of Stomatology, Capital Medical University, Beijing, China
The treatment of skeletal Class 111 malocclusion in adolescents is challenging. Maxillary protraction, particularly that using bone anchorage, has been proven to be an effective method for the stimulation of maxillary growth. However, the conventional procedure, which involves the surgical implantation of mini-plates, is traumatic and associated with a high risk. Three-dimensional (3D) digital technology offers the possibility of individualized treatment. Customized miniplates can be designed according to the shape of the maxillary surface and the positions of the roots on cone-beam computed tomography scans; this reduces both the surgical risk and patient trauma. Here we report a case involving a 12-year-old adolescent girl with skeletal Class 111 malocclusion and midface deficiency that was treated in two phases. In phase 1, rapid maxillary expansion and protraction were performed using 3D-printed mini-plates for anchorage. The mini-plates exhibited better adaptation to the bone contour, and titanium screw implantation was safer because of the customized design. The orthopedic force applied to each mini-plate was approximately $400-500 \mathrm{~g}$, and the plates remained stable during the maxillary protraction process, which exhibited efficacious orthopedic effects and significantly improved the facial profile and esthetics. In phase 2, fixed appliances were used for alignment and leveling of the maxillary and mandibular dentitions. The complete two-phase treatment lasted for 24 months. After 48 months of retention, the treatment outcomes remained stable.

[Korean J Orthod 2020;50(5):346-355]

Key words: Face mask, Implant design, Customized mini-plates, Threedimensional printing

Received July 17, 2019; Revised November 11, 2019; Accepted November 13, 2019.

Corresponding author: Yuxing Bai.

Professor, Dean, Department of Orthodontics, School of Stomatology, Capital Medical University, Tian Tan Xi Li \#4, Dongcheng District, Beijing 100050, China.

Tel +86-10-57099004 e-mail byuxing@ccmu.edu.cn

How to cite this article: Liang S, Xie X, Wang F, Chang Q, Wang H, Bai Y. Maxillary protraction using customized mini-plates for anchorage in an adolescent girl with skeletal Class 111 malocclusion. Korean J Orthod 2020;50:346-355.

(C) 2020 The Korean Association of Orthodontists.

This is an Open Access article distributed under the terms of the Creative Commons Attribution Non-Commercial License (http://creativecommons.org/licenses/by-nc/4.0) which permits unrestricted non-commercial use, distribution, and reproduction in any medium, provided the original work is properly cited. 


\section{INTRODUCTION}

Skeletal Class 111 malocclusion is relatively common, particularly in the Asian population. 'More than 50\% of patients with skeletal Class 111 malocclusion exhibit maxillary deficiency, ${ }^{2}$ which can be efficiently corrected by maxillary protraction in growing children. However, conventional maxillary protraction procedures that use the teeth and palate for anchorage can result in some dental effects such as increased proclination of the maxillary anterior teeth, increased retroclination of the mandibular anterior teeth, and extrusion and mesial drifting of the maxillary molars. ${ }^{3}$

Some studies have reported the use of mini-plate anchorage for better skeletal effects. ${ }^{4,5}$ However, for adaptation to the bony contour, conventional mini-plates need to be bent manually during the surgery. This leads to inaccuracy and increases the surgical duration. ${ }^{6}$ Moreover, the distance between the holes on conventional mini-plates is fixed, and it is difficult to determine 2-3 safe points for mini-screw insertion at a certain distance. In previous studies, mini-plates were usually implanted on the infrazygomatic crest or the lateral nasal wall, high above the root apices for the prevention of root damage. However, surgical visualization was poor and a large incision was required. ${ }^{7,8}$ Therefore, this procedure is complicated and traumatic and associated with a high risk.
Some researchers have studied the use of individualized titanium mini-plates for maxillary and mandibular orthognathic surgeries. ${ }^{9,10}$ However, there are few reports on the use of customized mini-plates in orthodontic treatment. Here we report a case involving a 12-year-old adolescent girl with skeletal Class 111 malocclusion and a concave facial profile that was treated in two phases. In phase 1, rapid maxillary expansion (RME) and protraction were performed using three-dimensional (3D)printed mini-plates for anchorage. The customized miniplates were designed such that they avoided the roots and precisely adapted to the bone contour. Thus, the risk associated with implant surgery was decreased. In phase 2, fixed appliances were used for alignment and leveling of the maxillary and mandibular dentitions.

\section{DIAGNOSIS AND ETIOLOGY}

A 12.5-year-old adolescent girl who experienced menarche at the age of 12 years presented with chief complaints of anterior crossbite and poor facial esthetics. She had no history of systemic disease and no family history of Class 111 characteristics. The patient's mother mentioned that her self-confidence was affected by her facial appearance. Extraoral examination revealed midface deficiency with a concave profile. Mandibular asymmetry was observed in the frontal view, with deviation of the mandibular midline to the right by $2 \mathrm{~mm}$.
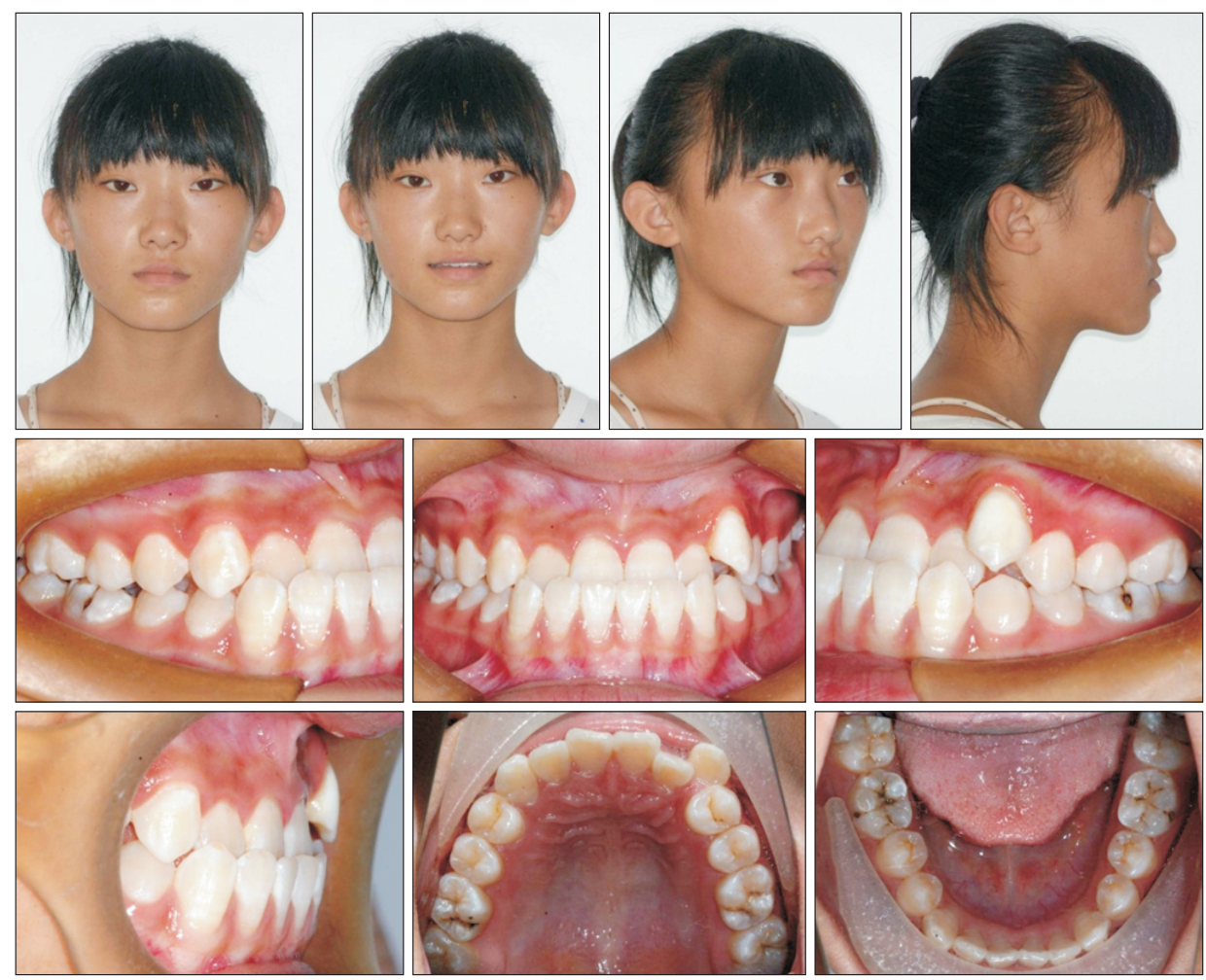

Figure 1. Pretreatment facial and intraoral photographs for an adolescent girl with skeletal Class III malocclusion and midface deficiency. 
Intraoral examination revealed Class 111 occlusion and anterior crossbite, which became an edge-to-edge bite on retrusion. Moderate crowding was present in the maxilla, and the left canine was displaced from its normal position. The mandibular midline was deviated to the right by $2 \mathrm{~mm}$ (Figure 1).

Dental models showed moderate crowding in the maxillary dentition, with a Class 111 occlusal relationship between the maxillary and mandibular teeth and anterior crossbite. A panoramic radiograph (Figure 2) showed that the alveolar bone height was normal, and that all four third molars were developing. There was a high-density shadow in the right maxillary sinus, which probably implied inflammation. A hand-wrist radiograph indicated a post-pubertal growth spurt.

Cephalometric analysis indicated skeletal Class 111 malocclusion with maxillary deficiency (ANB, $-3.5^{\circ}$; SNA, $75.9^{\circ}$; SNB, $79.4^{\circ}$ ). The mandibular plane angle was normal (FMA, 25.5 ). The maxillary anterior teeth were proclined (U1-SN angle, $114.0^{\circ}$ ) and the mandibular anterior teeth were retroclined (IMPA, $86.2^{\circ}$; Figure 2 and Table 1). Description of the measurements used in this study are depicited in Table 1.

\section{TREATMENT OBJECTIVES}

1. Stimulation of maxillary growth and moderation of
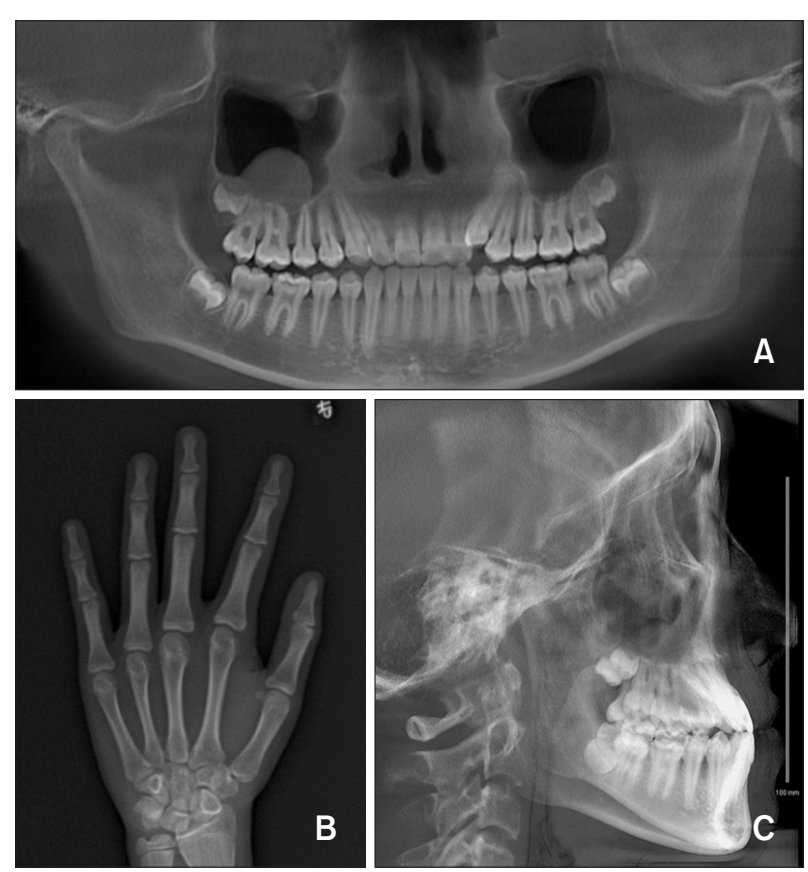

Figure 2. A pretreatment panoramic radiograph (A), hand-wrist radiograph (B), and lateral cephalogram (C) for an adolescent girl with skeletal Class III malocclusion and midface deficiency. the skeletal discrepancy

2. Elimination of the anterior crossbite and achievement of a Class 1 occlusion

3. Adjustment of the overjet and overbite to a normal range

4. Alignment and leveling of the maxillary and mandibular arches and adjustment of the midline

5. Achievement of an esthetic appearance by improving the concave facial profile

\section{TREATMENT ALTERNATIVES}

Considering the skeletal discrepancy, three treatment options were presented to the patient: fixed orthodontic treatment, combined orthodontic and orthognathic treatment, and orthopedic treatment. Fixed orthodontic treatment would not improve the lateral profile. Surgery was deemed inappropriate because the patient's mental health was affected by her appearance, and the patient and her parents were not willing to wait for a long period of time. Orthopedic treatment was likely to promote maxillary growth and improve the patient's lateral profile.

Conventional tooth-borne maxillary protraction has been recommended for the achievement of skeletal ef-

Table 1. Findings of lateral cephalogram analyses before and after facemask therapy for an adolescent girl with skeletal Class III malocclusion and midface deficiency

\begin{tabular}{lccc}
\hline Measurement & Pretreatment & $\begin{array}{c}\text { After } \\
\text { facemask } \\
\text { treatment }\end{array}$ & $\begin{array}{c}\text { Post- } \\
\text { treatment }\end{array}$ \\
\hline SNA $\left({ }^{\circ}\right)$ & 75.9 & 78.9 & 79.1 \\
SNB $\left(^{\circ}\right)$ & 79.4 & 81.1 & 81.5 \\
ANB $\left({ }^{\circ}\right)$ & -3.5 & -2.2 & -2.4 \\
A-Na perp $(\mathrm{mm})$ & -1.57 & 0.73 & 0.84 \\
FMA $\left({ }^{\circ}\right)$ & 25.5 & 25.0 & 25.1 \\
SN-GoGn $\left({ }^{\circ}\right)$ & 34.4 & 34.0 & 34.1 \\
PP-SN $\left(^{\circ}\right)$ & 12.2 & 14.3 & 13.08 \\
U1-PP $\left({ }^{\circ}\right)$ & 127.3 & 128.5 & 133.1 \\
$\mathrm{U} 1-S N\left({ }^{\circ}\right)$ & 114.0 & 117.0 & 121.2 \\
IMPA $\left({ }^{\circ}\right)$ & 86.2 & 86.5 & 81.1 \\
\hline
\end{tabular}

SNA, Sella-nasion-A point; SNB, sella-nasion-B point; ANB, A point-nasion-B point; A-Na perp, distance from A point to a line perpendicular to the Frankfort horizontal plane passing from the nasion point; FMA, Frankfort-mandibular plane angle; SN-GoGn, angle between sella-nasion and gonion-gnathion; PP-SN, angle between the palatal plane and sella-nasion; U1-PP, angle between the maxillary incisor and palatal plane; U1-SN, angle between the maxillary incisor and sella-nasion; IMPA, lower incisor to mandibular plane angle. 
fects in patients younger than 10 years. ${ }^{11}$ However, bone-anchored maxillary protraction (BAMP) is often applied after eruption of the maxillary permanent canines, which normally occurs at approximately 11-12 years of age, and it can result in remarkable skeletal changes. $^{7,8,12-15}$ Our patient was over 12 years of age. Moreover, the anterior teeth showed compensatory inclination and the maxillary dentition was very crowded. Therefore, we decided to perform maxillary protraction using mini-plates for anchorage.

Before implantation, we could clearly determine the bone thickness, bone density, and root positions on cone-beam computed tomography (CBCT) images. Accordingly, individualized mini-plates were designed such that they avoided the roots and precisely adapted to the bone contour. These customized mini-plates do not require manual bending and naturally adapt to the cortical bone surface, thus helping in reducing the surgical duration. Moreover, the distance between the holes on these plates is not fixed, and independent safe areas be- tween the roots can be chosen at a near or far distance. A lower implant position facilitates better visualization during surgery and requires only a small incision, which is nearly as long as the length of the mini-plate, under local anesthesia.

We anticipated that facemask therapy would decrease the maxillomandibular skeletal discrepancy. This therapy was approved by the Institutional Review Board (IRB) of Beijing Stomatological Hospital, Capital Medical University (IRB number: CMUSH-IRB-KJ-PJ-2017-04F) and the written informed consent was obtained from the patient. Because she was experiencing a post-pubertal growth spurt and had experienced menarche 6 months back, she had missed the best timing for facemask therapy. We explained to her parents that orthognathic treatment would be needed if maxillary growth could not be adequately stimulated or if maxillary growth was drastically exceeded by mandibular growth.

Following a discussion, the patient and her parents refused surgery and expressed a desire for quicker treat-
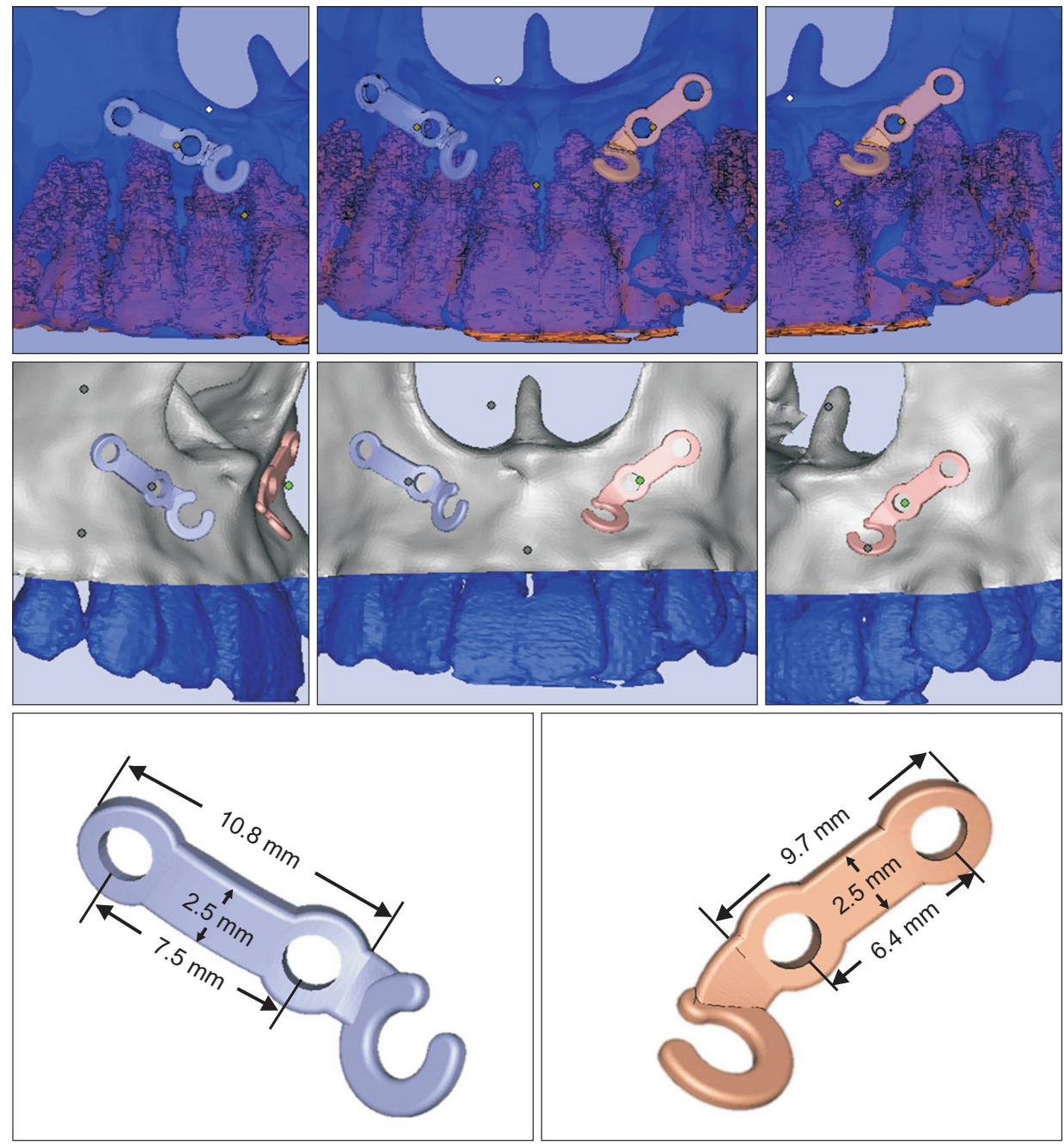

Figure 3. Design of customized titanium mini-plates for anchorage during facemask therapy for an adolescent girl with skeletal Class III malocclusion and midface deficiency. 
ment to improve her facial esthetics. Eventually, they opted for maxillary protraction as a more reliable treatment option and accepted the fact that she could require orthognathic surgery as she grew older.

\section{TREATMENT PROGRESS}

Preoperative CBCT was performed with the mandible in a retruded position.

In phase 1, treatment was initiated with RME using a Hass palatal expansion appliance for the achievement of a posterior buccal crossbite. The appliance was expanded twice a day $(0.25 \mathrm{~mm}$ per turn) for 14 days, following which it remained in the same position for 6 months for retention.

Facemask therapy was initiated after RME, at the age of 12 years and 9 months. Customized mini-plates were used for anchorage and designed by using 3-matic software ver. 11.0 (Materialise, Leuven, Belgium). The procedure was as follows. First, two safe points on both sides of the anterior segment of the maxilla were selected for implantation of the mini-screws. Second, the diameter and length of the mini-screws were determined. Third, the mini-plates were designed according to the shape of the bone surface, with holes at the selected safe positions. Fourth, a hook to receive the elastic was added above the lateral incisor on each side. The width and thickness of the mini-plates were 2.5 and $0.9 \mathrm{~mm}$, respectively, while the lengths of the right and left mini- plates were 10.8 and $9.7 \mathrm{~mm}$, respectively (Figure 3). Once they were designed, 3D printing was performed using the Concept Laser M2 device (Concept Laser, Lichtenfels, Germany) with titanium powder (Renishaw, Derbyshire, UK). The final plates were implanted in the anterior segment of the maxilla under local anesthesia. Each mini-plate was fixed with two titanium miniscrews $(6 \mathrm{~mm}$ in length, $1.7 \mathrm{~mm}$ in diameter; Synthes, Solothurn, Switzerland).

A 400-500-g orthopedic force was applied to each mini-plate, at an angle of $30^{\circ}$ from the occlusal plane such that the force passed through the center of resistance of the nasomaxillary complex. This would minimize counterclockwise rotation of the maxilla. The patient was required to wear the facemask for at least 12 hours a day. Facemask therapy was initiated 4 weeks after the implant surgery, and the anterior crossbite was corrected after 8 weeks of protraction. The overall duration of the facemask therapy was 10 months, when an acceptable profile and a Class 1 occlusal relationship were achieved (Figure 4). The mini-plates were then removed under local anesthesia. Cephalometric analysis (Figure 5 and Table 1) revealed sagittal growth of the maxilla. Maxillary growth was accelerated for improvement of the sagittal skeletal relationship. Meanwhile, the torques of the maxillary and mandibular incisors were maintained, maxillary molar extrusion was controlled, and maxillary arch crowding was relieved.

In phase 2, fixed orthodontic treatment was initiated
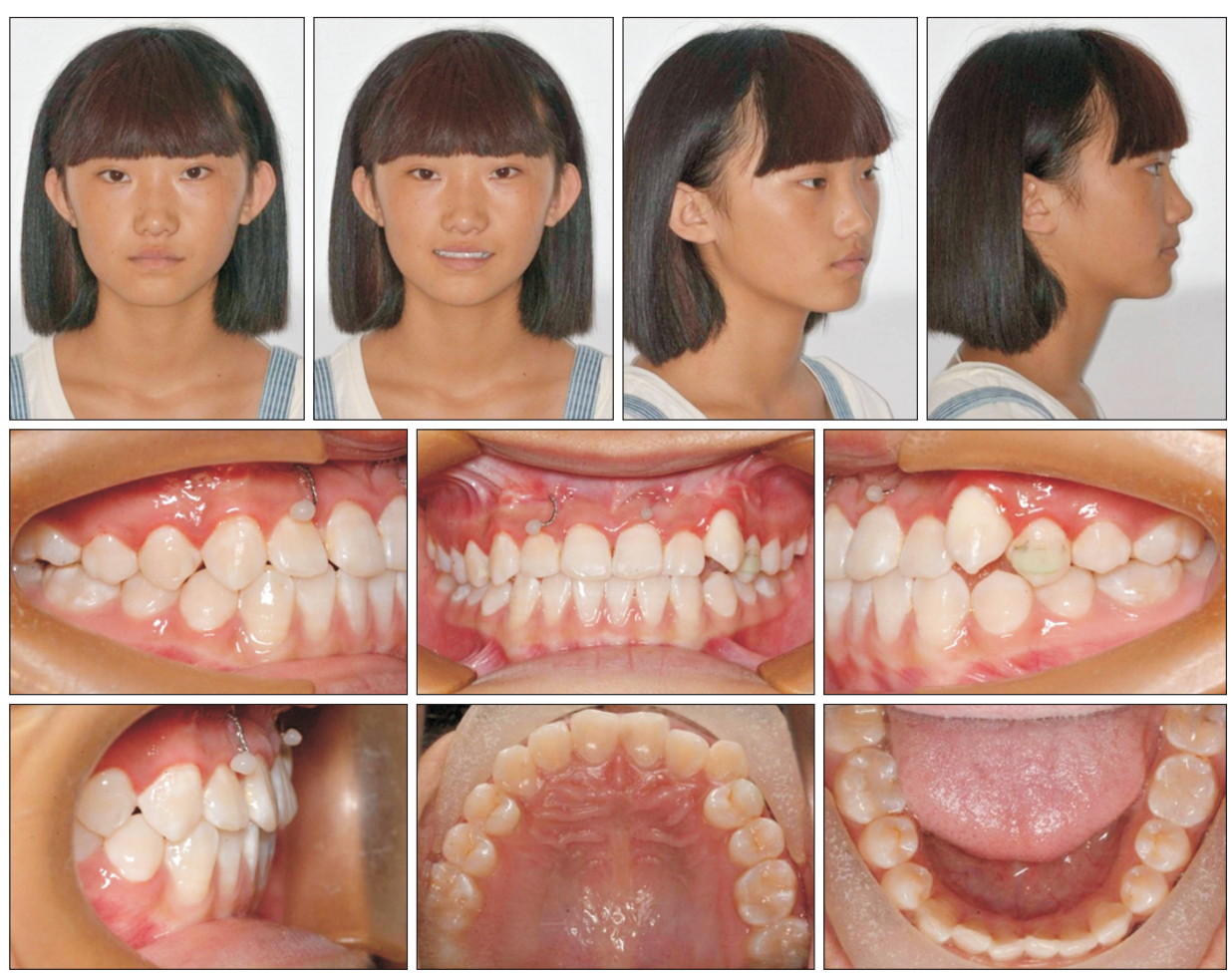

Figure 4. Facial and intraoral photographs obtained after 10 months of facemask therapy for an adolescent girl with skeletal Class III malocclusion and midface deficiency. An acceptable lateral profile and an overcorrected overjet have been achieved. The miniplates were subsequently removed. 
with the bonding of 0.022-inch brackets (MBT; 3M Unitek, Monrovia, CA, USA) in the maxillary and mandibular dentitions. Archwires were sequentially changed for alignment and leveling in both arches, and Class III elastics and interarch elastics were applied for adjustment of the occlusal relationship and mandibular midline. The fixed appliance treatment lasted for 12 months, follow-

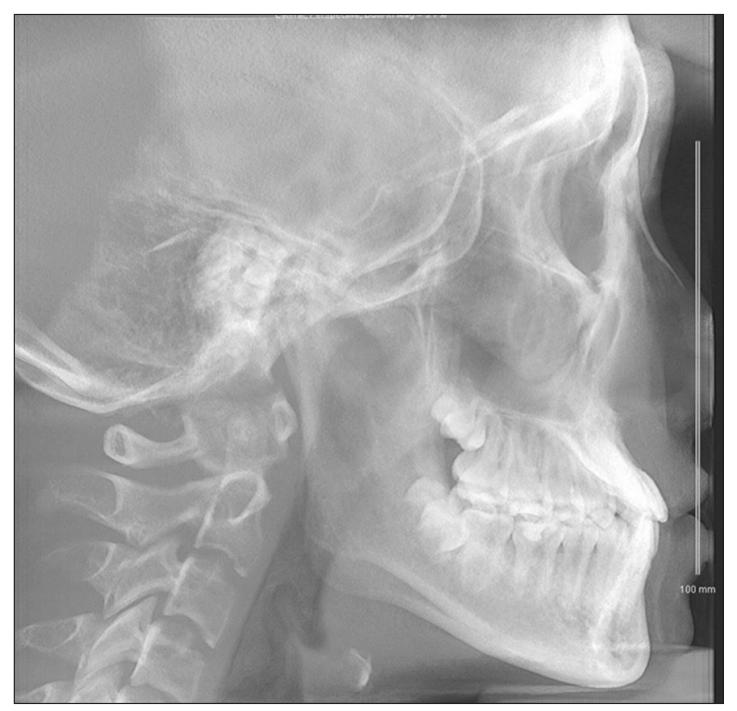

Figure 5. A lateral cephalogram obtained after facemask therapy for an adolescent girl with skeletal Class III malocclusion and midface deficiency. ing which the brackets were debonded. The patient was required to wear removable vacuum-formed retainers for more than 20 hours every day.

\section{RESULTS}

Following the comprehensive treatment, the patient achieved an esthetic lateral profile with ideal intercuspation, which were evident on post-treatment facial and intraoral photographs (Figure 6) and casts. Because of maxillary growth, the soft tissue profile became more convex, the upper lip protruded, and the lower lip retruded. At the same time, the anterior crossbite was eliminated and a Class 1 relationship was achieved between the maxillary and mandibular dentitions. The overbite and overjet also normalized. The improved facial and tooth appearances boosted the patient's confidence.

A post-treatment panoramic radiograph (Figure 7) indicated the absence of root resorption and alveolar bone loss, with satisfactory root parallelism. The round hyperdense lesion in the right maxillary sinus was still present.

The findings of cephalometric analysis (Figure 7 and Table 1) were consistent with those of the extraoral and intraoral examinations. In phase 1, the maxillomandibular skeletal discrepancy was resolved (SNA increased from $75.9^{\circ}$ to $78.9^{\circ}, \mathrm{A}-\mathrm{Na}$ perp increased from -1.57 to 0.73 $\mathrm{mm}$, A point moved forward, and ANB increased from $-3.5^{\circ}$ to $-2.2^{\circ}$ ) and the PP-SN angle increased from
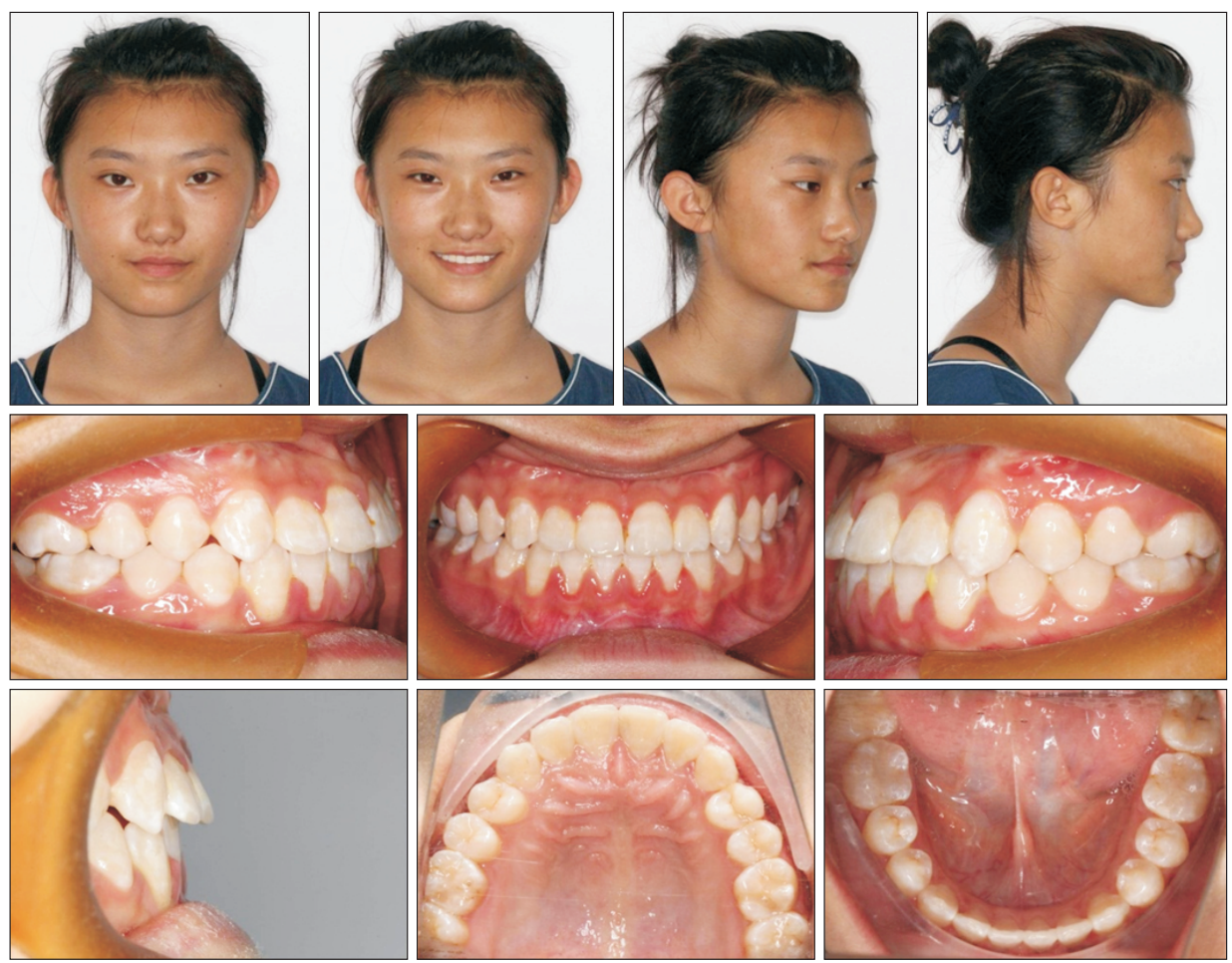

Figure 6. Post-treatment facial and intraoral photographs (12 months after facemask therapy) for an adolescent girl with skeletal Class III malocclusion and midface deficiency treated by facemask therapy and fixed appliance treatment. An improved lateral profile and a Class I occlusal relationship between the molars and canines can be observed. 

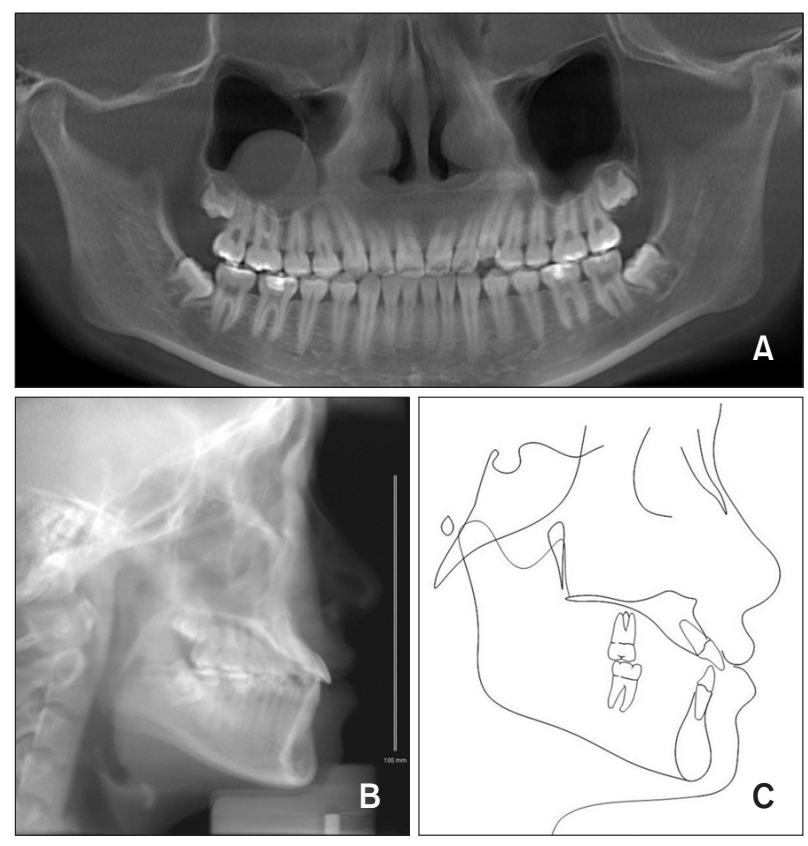

Figure 7. A post-treatment panoramic radiograph (A), lateral cephalogram (B) and superimposed cephalometric tracing (C) for an adolescent girl with skeletal Class III malocclusion and midface deficiency treated by facemask therapy and fixed appliance treatment. $12.2^{\circ}$ to $14.3^{\circ}$, indicating counterclockwise rotation of the maxilla. The torque of the maxillary incisors was slightly increased (U1-SN angle increased from $114.0^{\circ}$ to $117.0^{\circ}$ ), primarily because of counterclockwise rotation of the maxilla. The mandibular plane angle (FMA changed from $25.5^{\circ}$ to $25.0^{\circ}$ ) and the torque of the mandibular incisors were maintained (IMPA increased from $86.2^{\circ}$ to $86.5^{\circ}$ ). In phase 2 , the skeletal measurements remained stable (SNA increased from $78.9^{\circ}$ to $79.1^{\circ}$ and ANB decreased from $-2.2^{\circ}$ to $-2.4^{\circ}$ ) and the maxillary and mandibular incisors showed compensatory inclination (U1-SN angle increased from $117.0^{\circ}$ to $121.2^{\circ}$ while IMPA decreased from $86.5^{\circ}$ to $81.1^{\circ}$ ).

Superimposition of pre- and post-treatment cephalometric tracings facilitated the evaluation of improvements in the skeletal discrepancy and lateral soft tissue profile as well as the movements of the incisors and molars (Figure 8).

The most dramatic change was the significant improvement in the soft tissue profile. Along with forward movement of the upper lip, the zygomatic prominence and paranasal concavity also exhibited improvements.

From the post- and pretreatment CBCT images, we built a 3D color map of craniofacial hard tissue superimposed at the cranial base using Geomagic Qualify software ver. 2013 (Geomagic, Morrisville, NC, USA). The color bar ranged from $-3 \mathrm{~mm}$ (blue color) to $3 \mathrm{~mm}$ (red color), with red and yellow colors indicating outward

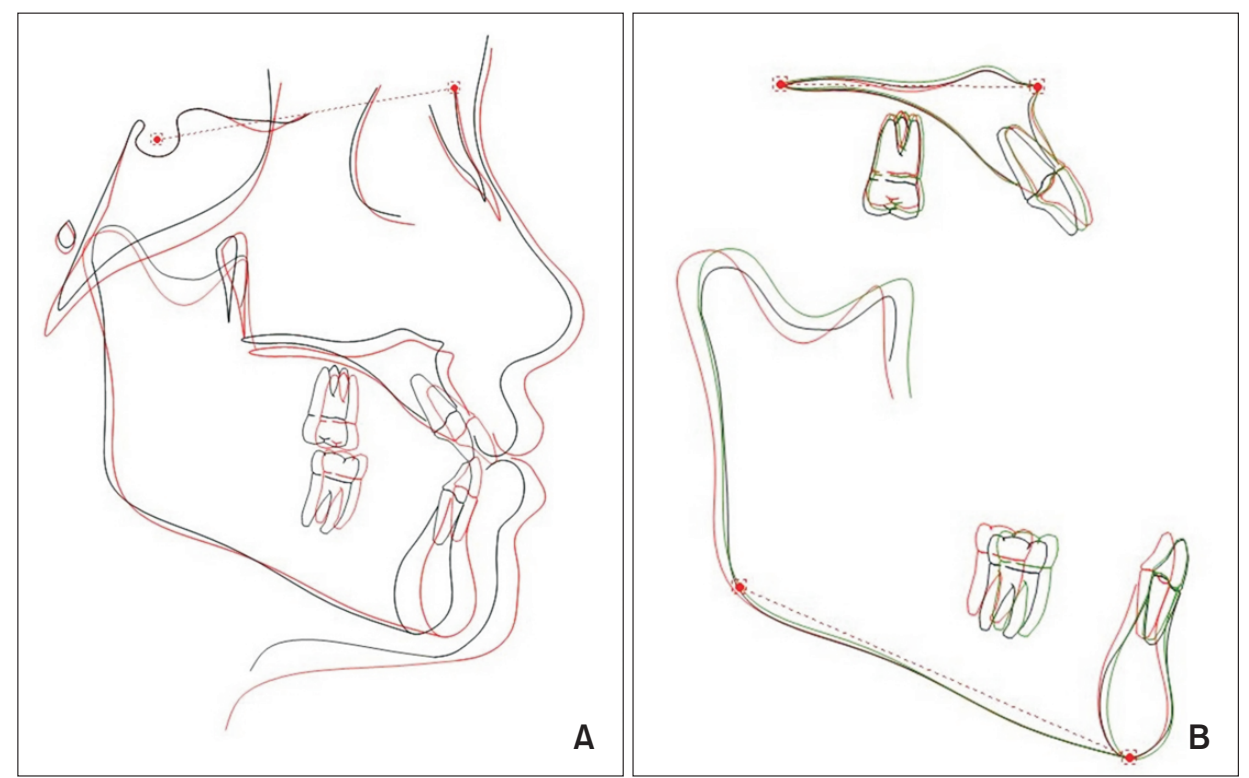

Figure 8. Superimpositions of pre- and post-treatment lateral cephalograms for an adolescent girl with skeletal Class III malocclusion and midface deficiency treated by facemask therapy and fixed appliance treatment. An improvement in the skeletal discrepancy and favorable changes in the lateral soft tissue profile can be observed. The movements of the incisors and molars can also be appreciated. A, Overall superimposition between pre- (black) and post treatment (red). B, Maxillary and mandibular superimposition between pre- (black), after facemask therapy (green), and post treatment (red). 


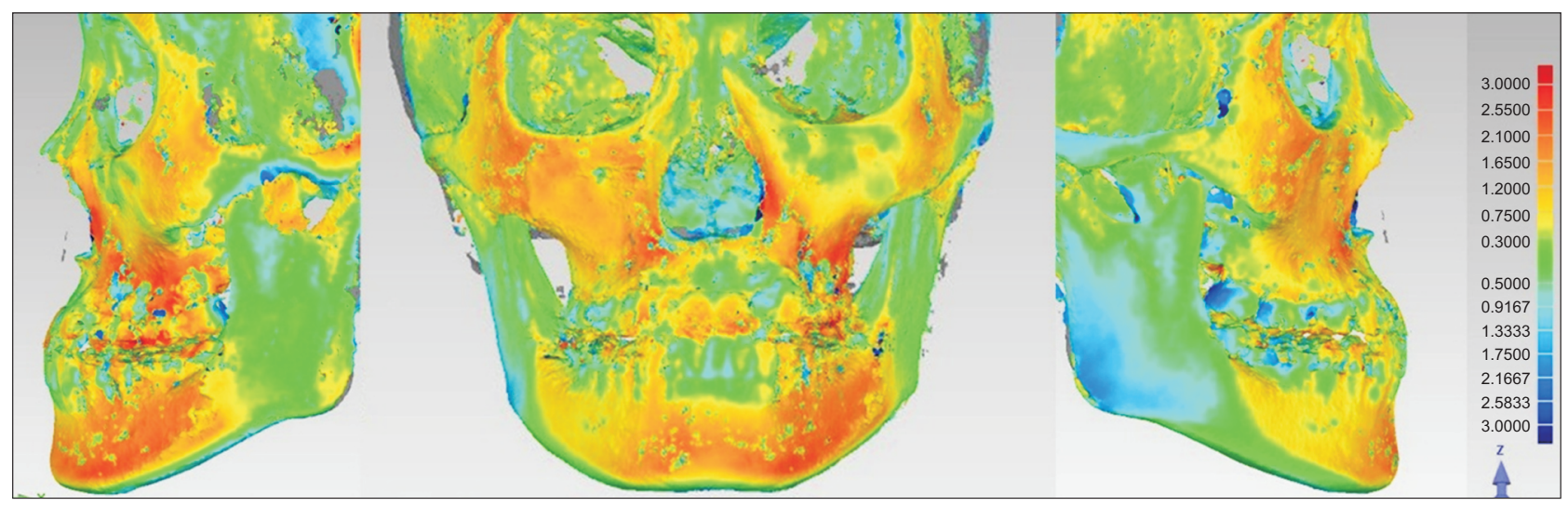

Figure 9. A three-dimensional color map (skeletal) generated by superimposition of pre- and post-treatment conebeam computed tomography images for an adolescent girl with skeletal Class III malocclusion and midface deficiency treated by facemask therapy and fixed appliance treatment. There is significant maxillary growth (T1, before treatment; T2, after treatment). The red and yellow areas indicate outward movements of the T2 skeletal surfaces in relation to the T1 surfaces, the blue areas indicate inward movements of the T2 surfaces relative to the T1 surfaces, and the green areas represent little or no change.
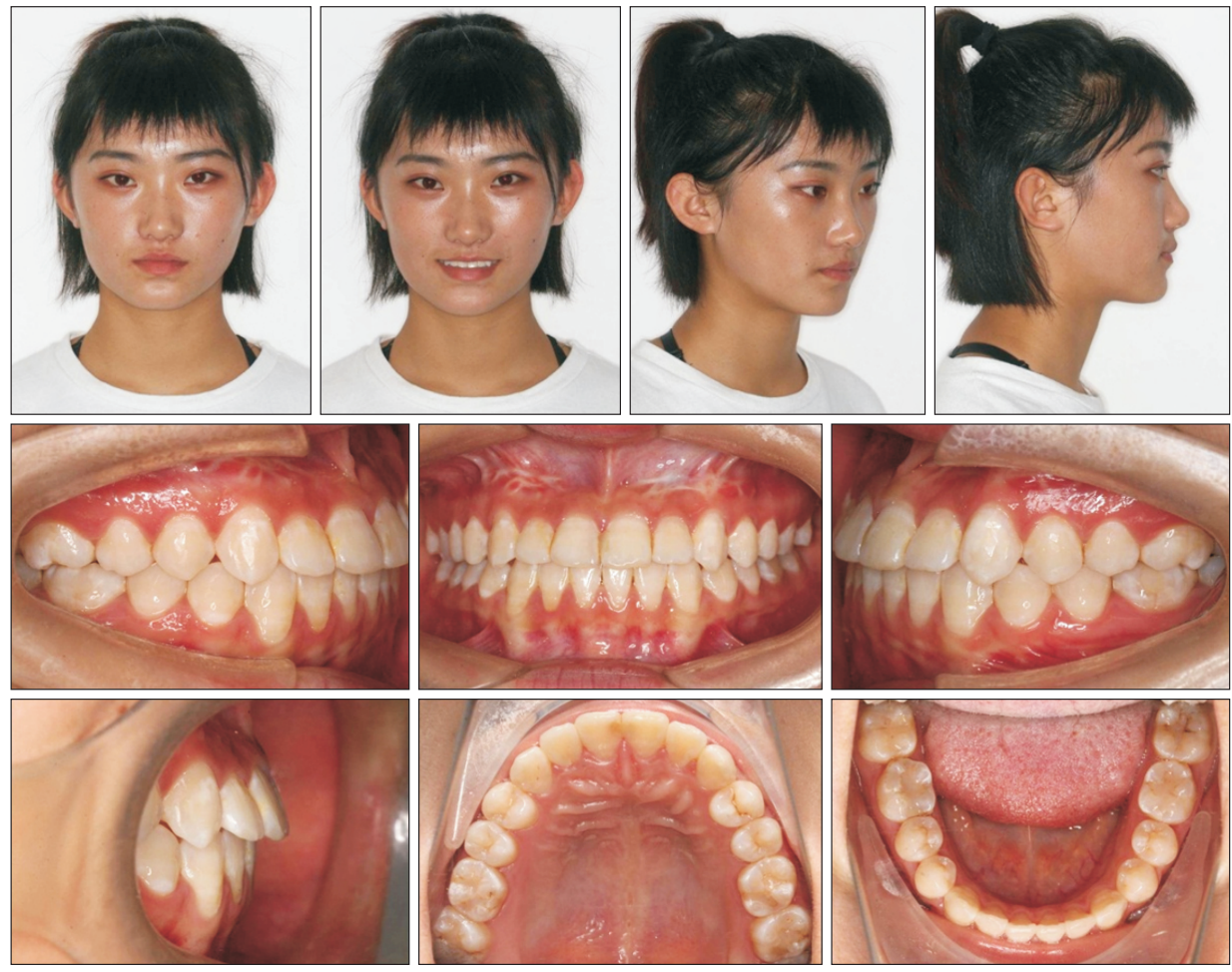

Figure 10. Post-retention photographs obtained 48 months after debonding for an adolescent girl with skeletal Class III malocclusion and midface deficiency treated by facemask therapy and fixed appliance treatment. The occlusal relationship and profile are stable. movements of the after treatment (T2) skeletal surfaces in relation to the before treatment (T1) surfaces, blue color indicating inward movement of the T2 surfaces in relation to the $\mathrm{T} 1$ surfaces, and green color indicating no change. The color maps showed significant growth of the maxilla and zygoma (Figure 9), consistent with the soft tissue changes. The color maps also showed significant forward mandibular displacement, which oc- curred because of mandibular positioning to an anterior edge-to-edge bite during pretreatment CBCT taking (Figure 2).

After 48 months of retention, the occlusal relationship, overbite, and overjet were stable and the facial profile was satisfactory (Figure 10). 


\section{DISCUSSION}

It is difficult to obtain a good profile in patients with skeletal Class 111 anomalies who are experiencing postpubertal growth spurts by orthodontic treatment alone. Many studies have demonstrated that the skeletal effects of facemask therapy with mini-plate anchorage are superior to those of conventional facemask therapy, with the former minimizing undesired side effects such as dental changes and clockwise rotation of the mandible. $^{7,8,12-15}$ To increase the orthopedic effect, we used skeletal anchorage that directly transferred orthopedic forces to the maxilla in our case. The maxilla successfully moved forward while the maxillomandibular skeletal discrepancy was alleviated. The torque of the maxillary incisors was slightly increased and the mandibular plane angle was maintained.

Although BAMP has minimal dental effects, it cannot completely avoid dental changes. Previous studies showed that maxillary protraction with mini-plate anchorage resulted in inclination of the maxillary anterior teeth, although the degree of change was smaller than that with tooth-borne maxillary protraction. ${ }^{12-14}$ In the present case, after phase 1 treatment, the torque of the maxillary incisors was slightly increased, primarily because of counterclockwise rotation of the maxilla. Moreover, there was contact between the maxillary and mandibular anterior teeth during the process of anterior crossbite correction, and the mandibular incisors exerted a forward and upward thrust on the maxillary incisors, resulting in inclination of the latter. In phase 2 treatment, the maxillary incisors were further inclined for alignment of the crowded arch.

Mini-plates are widely used for anchorage in BAMP. ${ }^{12-15}$ However, conventional mini-plates need to be bent manually during the procedure for adaptation to the bony contour, which leads to inaccuracy, and the implant location is often determined during the procedure. ${ }^{6}$ This increases the surgical duration, which is associated with an increased risk of infection and postoperative pain. Individualized titanium mini-plates are reportedly used in orthognathic surgery for the prevention of damage to the mandibular nerve and achievement of more accurate outcomes. ${ }^{9,10}$ In the present case, we used customized mini-plates that do not require manual bending and naturally adapt to the cortical bone surface. The implant position is determined before the procedure, which considerably reduces the surgical duration. Moreover, the distance between the holes on customized mini-plates is not fixed, as mentioned earlier. Therefore, individualized mini-plates allow for simplification of the surgical procedure and reduce the duration and risk of the surgery.

The timing of facemask therapy is crucial. Delaire ${ }^{16}$ suggested that facemask therapy should be initiated be- fore 8 years of age. Meanwhile, it has been recognized that skeletal changes in the maxilla are easier to achieve before the age of 10 years. ${ }^{11}$ However, maxillary protraction with skeletal anchorage is often applied after eruption of the maxillary permanent canines at an approximate age of 11-12 years. Previous studies have reported forward movement of the maxilla by treatment based on skeletal anchorage in patients with a mean age of 11.2 years. ${ }^{7,13}$ De Clerck et al. ${ }^{17}$ proposed a BAMP procedure that used bone-anchored Class 111 traction and achieved a favorable maxillary growth response in patients with a mean age of 11.1 years. Subsequently, studies showed orthopedic changes after BAMP treatment in patients with a mean age of $11.75-11.9$ years. ${ }^{8,14}$ In a case report, Esenlik et al. ${ }^{15}$ documented skeletal effects in a patient aged 14.4 years. Our patient, who began facemask therapy at 12 years and 9 months of age (after the pubertal growth spurt), missed the optimal timing for facemask treatment initiation but still achieved a good outcome. The SNA angle increased by $3^{\circ}$ and point A moved forward by $2.3 \mathrm{~mm}$. This significant improvement was due to forward movement of the maxilla, which could be confirmed by superimpositions of CBCT images. This change also caused a remarkable change in her lateral profile.

RME before maxillary protraction can be beneficial for patients with skeletal Class 111 malocclusion because it can reduce the midfacial concavity. ${ }^{18}$ It has been suggested that displacement of the craniofacial structures is more favorable when maxillary protraction is performed with RME than when it is performed without RME. ${ }^{19}$ Therefore, RME was performed before maxillary protraction in our patient, and it may have contributed to the overall outcome.

The 3D digital technology has been widely used in the field of orthodontics. It greatly improves the accuracy of diagnosis and treatment and offers the possibility of individualized treatment. ${ }^{20}$ In the present case, 3D images of the teeth, maxillary bone, and maxillary sinus were acquired using $\mathrm{CBCT}$, which facilitated determination of the insertion sites and designing of the customized mini-plates. The 3D printing technique directly manufactures 3D products on the basis of a computer-aided design file. Titanium is a biologically compatible metal and widely used in the fields of medicine and dentistry. We used titanium powder (Renishaw) to print the miniplates for our patient.

\section{CONCLUSION}

In conclusion, we described the successful use of customized mini-plates for anchorage during facemask therapy in an adolescent girl with skeletal Class 111 malocclusion and midface deficiency. BAMP moderated 
the maxillomandibular discrepancies, restricted dental compensation, and achieved remarkable improvements in the occlusal relationship and lateral profile. The findings from this case indicate that the clinical application of customized mini-plates is worth promoting because of advantages, such as a small incision, individualized insertion points, an easy surgical procedure, and a short surgical duration.

\section{CONFLICTS OF INTEREST}

No potential conflict of interest relevant to this article was reported.

\section{ACKNOWLEDGEMENTS}

This study was supported by Beijing Municipal Administration of Hospital Clinical Medicine Development of Special Funding Support (ZYLX201703), Beijing Municipal Administration of Hospitals' Ascent Plan (DFL20151401), and Beijing Municipal Science and Technology Commission (Z151100003915137).

\section{REFERENCES}

1. Allwright WC, Bundred WH. A survey of handicapping dentofacial anomalies among Chinese in Hong Kong. Int Dent J 1964;14:505-19.

2. Guyer EC, Ellis EE 3rd, McNamara JA Jr, Behrents RG. Components of class 111 malocclusion in juveniles and adolescents. Angle Orthod 1986;56:7-30.

3. Kim JH, Viana MA, Graber TM, Omerza FF, BeGole EA. The effectiveness of protraction face mask therapy: a meta-analysis. Am J Orthod Dentofacial Orthop 1999;115:675-85.

4. De Clerck HJ, Cornelis MA, Cevidanes LH, Heymann GC, Tulloch CJ. Orthopedic traction of the maxilla with miniplates: a new perspective for treatment of midface deficiency. J Oral Maxillofac Surg 2009;67:2123-9.

5. Kook YA, Bayome M, Park JH, Kim KB, Kim SH, Chung KR. New approach of maxillary protraction using modified C-palatal plates in Class 111 patients. Korean J Orthod 2015;45:209-14.

6. Cornelis MA, Scheffler NR, Mahy P, Siciliano S, De Clerck HJ, Tulloch JF. Modified miniplates for temporary skeletal anchorage in orthodontics: placement and removal surgeries. J Oral Maxillofac Surg 2008;66:1439-45.

7. Sar C, Arman-Özçırpıcı A, Uçkan S, Yazıcı AC. Comparative evaluation of maxillary protraction with or without skeletal anchorage. Am J Orthod Dentofacial Orthop 2011;139:636-49.

8. Hino CT, Cevidanes LH, Nguyen TT, De Clerck HJ,
Franchi L, McNamara JA Jr. Three-dimensional analysis of maxillary changes associated with facemask and rapid maxillary expansion compared with bone anchored maxillary protraction. Am J Orthod Dentofacial Orthop 2013;144:705-14.

9. Suojanen J, Leikola J, Stoor P. The use of patientspecific implants in orthognathic surgery: a series of 32 maxillary osteotomy patients. J Craniomaxillofac Surg 2016;44:1913-6.

10. Brunso J, Franco M, Constantinescu T, Barbier L, Santamaría JA, Alvarez J. Custom-machined miniplates and bone-supported guides for orthognathic surgery: a new surgical procedure. J Oral Maxillofac Surg 2016;74:1061.e1-1061.e12.

11. Saadia M, Torres E. Sagittal changes after maxillary protraction with expansion in class 111 patients in the primary, mixed, and late mixed dentitions: a longitudinal retrospective study. Am J Orthod Dentofacial Orthop 2000;117:669-80.

12. Cha BK, Ngan PW. Skeletal anchorage for orthopedic correction of growing class 111 patients. Semin Orthod 2011;17:124-37.

13. Lee NK, Yang IH, Baek SH. The short-term treatment effects of face mask therapy in Class 111 patients based on the anchorage device: miniplates vs rapid maxillary expansion. Angle Orthod 2012;82:846-52.

14. Ağlarcı C, Esenlik E, Findık Y. Comparison of shortterm effects between face mask and skeletal anchorage therapy with intermaxillary elastics in patients with maxillary retrognathia. Eur J Orthod 2016;38:313-23.

15. Esenlik E, Ağlarcı C, Albayrak GE, Fındık Y. Maxillary protraction using skeletal anchorage and intermaxillary elastics in Skeletal Class 111 patients. Korean J Orthod 2015;45:95-101.

16. Delaire J. Maxillary development revisited: relevance to the orthopaedic treatment of Class 111 malocclusions. Eur J Orthod 1997;19:289-311.

17. De Clerck H, Cevidanes L, Baccetti T. Dentofacial effects of bone-anchored maxillary protraction: a controlled study of consecutively treated Class 111 patients. Am J Orthod Dentofacial Orthop 2010;138: 577-81.

18. Kiliç N, Kiki A, Oktay H, Erdem A. Effects of rapid maxillary expansion on Holdaway soft tissue measurements. Eur J Orthod 2008;30:239-43.

19. Gautam P, Valiathan A, Adhikari R. Skeletal response to maxillary protraction with and without maxillary expansion: a finite element study. Am J Orthod Dentofacial Orthop 2009;135:723-8.

20. Bai YX. [Application of three-dimensional digital technology in the diagnosis and treatment planning in orthodontics]. Zhonghua Kou Qiang Yi Xue Za Zhi 2016;51:326-30. Chinese. 